\title{
Resonant and nonresonant $x$-ray scattering spectra of some poly(phenylenevinylene)s
}

\author{
J.-H. Guo, Martin Magnuson, C. Såthe, J. Nordgren, L. Yang, Y. Luo, H. Ågren, K. Z. Xing, \\ N. Johansson, William R. Salaneck, R. Daik and W. J. Feast
}

\section{Post Print}

N.B.: When citing this work, cite the original article.

Original Publication:

J.-H. Guo, Martin Magnuson, C. Såthe, J. Nordgren, L. Yang, Y. Luo, H. Ågren, K. Z. Xing, N. Johansson, William R. Salaneck, R. Daik and W. J. Feast, Resonant and nonresonant X-ray scattering spectra of some poly(phenylenevinylene)s, 1998, Journal of Chemical Physics, (108), 14, 5990-5996.

http://dx.doi.org/10.1063/1.476011

Copyright: American Institute of Physics (AIP) http://www.aip.org/

Postprint available at: Linköping University Electronic Press

http://urn.kb.se/resolve?urn=urn:nbn:se:liu:diva-54988 


\title{
Resonant and nonresonant $x$-ray scattering spectra of some poly(phenylenevinylene)s
}

\author{
J.-H. Guo, M. Magnuson, C. Såthe, and J. Nordgren \\ Department of Physics, Uppsala University, Box 530, S-75121 Uppsala, Sweden \\ L. Yang, Y. Luo, and H. Ågren \\ Computational Physics, Institute of Physics and Measurement Technology, Linköping University, \\ S-58183, Linköping, Sweden
}

K. Z. Xing, N. Johansson, and W. R. Salaneck

Surface Physics and Chemistry, Institute of Physics and Measurement Technology, Linköping University, S-58183, Linköping, Sweden

R. Daik and W. J. Feast

IRC in polymer Science and Technology, Durham University South Road, Durham DH1 3LE, United Kingdom

(Received 18 July 1997; accepted 8 January 1998)

\begin{abstract}
The electronic structure of some poly(phenylenevinylene)s have been investigated by resonant and nonresonant x-ray inelastic scattering spectroscopies. The nonresonant as well as all resonant spectra for each polymer demonstrate benzene-like features, indicating a local character of the x-ray emission in which the phenyl ring acts as a building block. Theoretical simulations of $\mathrm{x}$-ray energies and intensities taking the repeat unit as a model molecule of the polymer agree with the experimental spectra fairly well. The edges of the occupied bands have been identified in the nonresonant spectra of each polymer. By subtracting the emission energy of the highest occupied molecular orbital in the nonresonant spectrum from the core excitation energy in the resonant spectrum an alternative way to determine the optical band gap is obtained. As for free benzene the outer $\pi$ band in the polymer spectra show a depletion of the emission going from the nonresonant to the resonant $\mathrm{x}$-ray emission spectra. It is demonstrated that this transition, which is strictly symmetry forbidden for free benzene, becomes effectively forbidden in the polymer case as a result of strong interference effects, and it is argued that this is the general case for resonant x-ray emission of conjugated polymers as far as the frozen orbital approximation holds. (C) 1998 American Institute of Physics. [S0021-9606(98)50914-2]
\end{abstract}

\section{INTRODUCTION}

Organic conjugated polymers have the electronic structure of semiconductors and can be doped to become good conductors. ${ }^{1}$ Conjugated polymers are now used as active materials in a wide variety of prototype applications such as light emitting diodes ${ }^{2}$ and organic transistors. ${ }^{3,4}$ As a first stage of an investigation it is important to obtain insight into the electronic properties of the undoped neutral compounds and to determine the characteristics of hole injection processes and light emission in connection with luminescence phenomena. Most of the interesting chemistry and physics of conjugated polymers is associated with the details of the electronic structure at the valence and conduction band edges and, in this connection, various electron spectroscopies can be used as tools for diagnosis of the relevant electronic and geometric properties.

Electron spectra often show a complicationsimplification pattern with respect to the size of an oligomer. The simple spectral structure of the free monomer is complicated by the additional repeat units. However, a simplification can take place when periodic boundary conditions are introduced, with spectra that might show remarkable molecular-like features. The convergence pattern is evidently dependent on the type of electronic process, and on the type and size of the repeat unit. Core photoelectron spectra (XPS) are believed to converge quite rapidly along an oligomer series due to the local nature of the core excitation/ deexcitation processes, which has been demonstrated previously by x-ray absorption (XA) spectroscopy. However, little seems to be known experimentally about the corresponding behavior for radiative and nonradiative emission spectroscopies in oligomers and polymers. This paper describes a study of a set of poly(phenylenevinylene)s using x-ray emission (XE) spectroscopy, which has been shown to be a powerful technique for studying electronic structures in other contexts.

$\mathrm{X}$-ray emission spectra reflect resonant and nonresonant $\mathrm{x}$-ray scattering which occur due to an interaction of incident $\mathrm{x}$-ray photons with a target consisting of atoms, molecules or a solid. The target is excited from the ground state $|o\rangle$ to a core excited state $|i\rangle$ by absorption of an incoming x-ray photon $(\gamma)$ with specific frequency, wave vector and polarization vector. The core excited state is metastable due to vacuum zero vibrations or interelectron Coulomb interaction and can therefore decay to final states $|f\rangle$ in two different ways, by emitting $\mathrm{x}$-ray photons or by emitting high-energy 


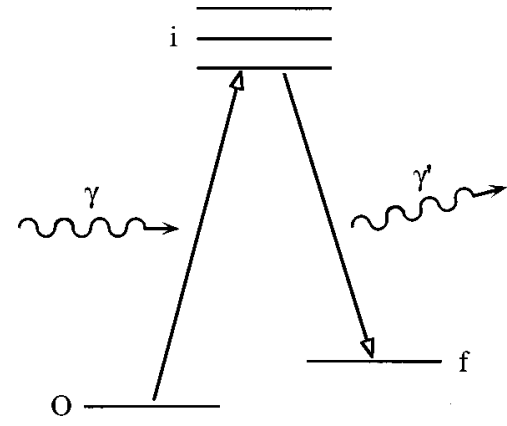

FIG. 1. Schematic diagram of the resonant x-ray Raman process.

Auger electrons; $M+\gamma \rightarrow M_{i} \rightarrow M_{f}+\gamma^{\prime}$ and $M+\gamma \rightarrow M_{i}$ $\rightarrow M_{f}+e^{-}$constituting the radiative, respectively nonradiative, decay channels of the $\mathrm{x}$-ray scattering process. When the frequency of the incident $x$-ray photons is tuned below or closely above the core ionization threshold resonant core excitation takes place. It is natural to refer to this case as to resonant x-ray scattering (RXS) or x-ray Raman scattering. The absorption-emission processes and the energy relations are sketched in Fig. 1 and is further described in the method and computational section of this paper.

The x-ray emission technique provides a means of extracting chemical information in the form of molecular orbital (MO) population and local density distribution of certain symmetries owing to the dipole character of the radiative decay and the localization of core-hole states. ${ }^{5,6}$ The resonance inelastic $\mathrm{x}$-ray scattering (RIXS) measurements are symmetry selective at high resolution. However, the relatively low fluorescence yield and instrument efficiencies associated with $\mathrm{x}$-ray emission in the ultra-soft $\mathrm{x}$-ray regime results in a considerable demand on the experimental measurements in this spectral region. Therefore, an intense excitation source is required, something that has limited the activity in this field.

In the present work we probe the electronic structure of a set of poly(phenylenevinylene)s (see in Fig. 2): poly(phenylenevinylene) (PPV), poly $\left(4,4^{\prime}\right.$-diphenylene diphenylvinylene) (PDPV) and, poly(1,3phenylenediphenylvinylene) (PMPV). We use $\mathrm{x}$-ray absorp-

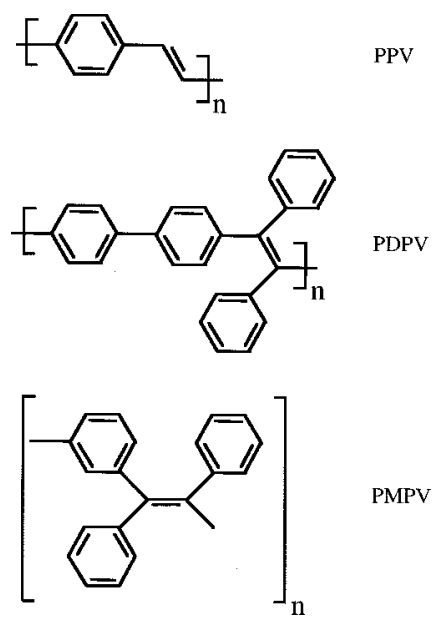

FIG. 2. Schematic diagrams of poly-phenylene-vinylene compounds. tion and x-ray emission spectroscopies in order to understand the influence of alkyl substituents in the top region of the valence band and at the band gap. These compounds are made up of six membered carbocyclic aromatic rings connected by short hydrocarbon bridges. We have investigated the general character of the polymers in both resonant and nonresonant inelastic x-ray scattering spectra. We find indeed a strong molecular-like appearance of the polymer spectra. This appearance is not a signature of the repeat unit but of the benzene ring, which thus has a large contribution in the spectra. To compare our experimental findings with theory we have carried out calculations of the nonresonant and resonant spectra within the molecular orbital model to investigate the possibility of a building block interpretation for $\mathrm{x}$-ray emission.

\section{EXPERIMENT}

The experiments were performed at beamline 7.0 at the Advanced Light Source, Lawrence Berkeley National Laboratory (LBNL). The beamline comprises a 99-pole, $5 \mathrm{~cm}$ period undulator and a spherical-grating monochromator ${ }^{7}$ covering the spectral energy range between 60 and $1000 \mathrm{eV}$. Near edge $\mathrm{x}$-ray absorption fine structure (NEXAFS) spectra were obtained by measuring the total electron yield from the sample with $0.15 \mathrm{eV}$ resolution of the monochromator of the beamline. The NEXAFS spectra were normalized to the incident photon current using a clean gold mesh in front of the sample to correct for intensity fluctuations in the photon beam. The XE spectra were recorded using a high-resolution grazing-incidence $\mathrm{X}$-ray fluorescence spectrometer. ${ }^{8}$ During the $\mathrm{x}$-ray emission measurements the resolution of the monochromator of the beamline and the x-ray fluorescence spectrometer were $0.15 \mathrm{eV}$ and $0.50 \mathrm{eV}$, respectively. The samples were scanned during the recording of XE spectra to avoid the effects from photon-induced decomposition of the polymer molecules. The base pressure in the experimental chamber was $5 \times 10^{-9}$ mbar.

The PPV sample was prepared from a soluble THTleaving group precursor polymer. ${ }^{9}$ The precursor was spincoated onto optically flat $\mathrm{Si}(110)$ wafers, and converted to PPV at $240{ }^{\circ} \mathrm{C}$ for 10 hours under a mild vacuum of $5 \times 10^{-6}$ mbar. The PDPV and PMPV samples were prepared by McMurry coupling of 4,4'-dibenzoylbiphenyl and metadibenzoylbenzene respectively, following the method described by Millichamp. ${ }^{10}$ The crude products were purified by equilibrium fractionation (chloroform solven/heptane non-solvent @20 ${ }^{\circ} \mathrm{C}$ ) following the technique described previously. ${ }^{11}$ The PMPV and PDPV polymers were spincoated from $2 \mathrm{mg} / \mathrm{ml}$ polymer/chloroform solution onto optically flat $\mathrm{Si}(110)$ substrates. All the samples were sealed in an $\mathrm{N}_{2}$ atmosphere in glass and were exposed to air for very short time just before they were introduced into the vacuum system for XE measurements. 


\section{THEORETICAL METHOD}

The present calculation assumes second order perturbation theory for the interaction of $\mathrm{x}$-ray photons with the target. The cross section of resonant $\mathrm{x}$-ray inelastic scattering (RIXS) is then expressed as: ${ }^{12-15}$

$$
\sigma\left(\omega^{\prime}, \omega_{o}\right)=\sum_{\nu n} \frac{\omega^{\prime}}{\omega}\left|F_{\nu n}(\omega)\right|^{2} \Phi\left(\omega^{\prime}+\omega_{\nu n}-\omega_{o}\right),
$$

where $\Phi\left(\omega-\omega_{o}\right)$ in the incoming photon distribution function centered at frequency $\omega_{o} .\left|F_{\nu n}(\omega)\right|$ is the KramersHeisenberg formula for the resonant $\mathrm{x}$-ray scattering amplitude ${ }^{16,17}$

$$
\begin{aligned}
& F_{\nu n}(\omega)=\sum_{k} f_{\nu n}^{k}(\omega), \\
& f_{\nu n}^{k}(\omega)=\alpha \omega_{\nu k} \omega_{n k}(\nu) \frac{\left(\mathbf{e}_{1}^{*} \mathbf{d}_{\nu k}\right)\left(\mathbf{e}_{2} \mathbf{d}_{k n}(\nu)\right)}{\omega-\omega_{\nu k}+i \Gamma_{\nu k}},
\end{aligned}
$$

where $f_{\nu n}^{k}(\omega)$ denotes the partial or channel amplitude of the RIXS process. The $k$ th channel amplitude $f_{\nu n}^{k}(\omega)$ describes the two-photon process of absorption of incoming and emission of final x-ray photons, the frequencies and polarization vectors of which are $\omega, \omega^{\prime}$ and $\mathbf{e}_{1}, \mathbf{e}_{2}$, respectively. The index $k$ enumerates localized or delocalized core orbitals $\psi_{k}$. (atomic units are here used $h=m=e=1, \alpha=1 / 137$ ). The following notations are assumed $\omega_{\nu k}=E\left(k^{-1} \nu\right)-E_{o}$, $\omega_{n k}(\nu)=E\left(k^{-1} \nu\right)-E\left(n^{-1} \nu\right), \quad \mathbf{d}_{\nu k}=\left\langle 0|\mathbf{d}| k^{-1} \nu\right\rangle \quad$ and $\mathbf{d}_{k n}(\nu)=\left\langle k^{-1} \nu|\mathbf{d}| n^{-1} \nu\right\rangle$ are resonant frequencies and dipole matrix elements of x-ray absorption $(k \rightarrow \nu)$ and emission $(n \rightarrow k)$ transitions, respectively. $\omega_{\nu n}=E\left(n^{-1} \nu\right)-E_{o}$ is a frequency for the optical excitation $n \rightarrow \nu$ and is equal to the difference between energies $E\left(n^{-1} \nu\right)$ and $E_{o}$ of excited $\left|n^{-1} \nu\right\rangle$ and ground $|0\rangle$ molecular states; $\Gamma_{\nu k}$ is the half width at half maximum (HWHM) of the x-ray absorption line $k \rightarrow \nu$. For the randomly oriented gas or solvent molecules, orientational averaging should be conducted. A detailed description of this referring to the case of randomly oriented molecules can be found in Ref. 18. The range of validity of the Kramers-Heisenberg formula (2) has been discussed in, for example, Ref. 12.

The nonresonant $x$-ray emission spectra are obtained by having the energy of the bombarding particles transcend the core ionization threshold. In this situation the x-ray emission spectral profile is practically independent of the excitation energy and the excitation agency (photon, electron or heavy particle). Owing to this fact the spectral shape of $x$-ray emission has been described often using a two-step model with the emission decoupled from the excitation. By contrast, the shapes of resonant $\mathrm{x}$-ray emission spectra are dependent on the way of preparation of the core excited state of the target, and with use of energy tunable photon sources, the $\mathrm{x}$-ray emission spectra are observed to be strongly frequency dependent when the excitation frequency approaches the x-ray photo-absorption threshold. The accompanying theoretical descriptions must then switch from a two-step to a one-step model (Eqs. 1, 2, 3), thus with the excitation and the deexcitation treated as one, nonseparable, scattering event.
A major feature of the resonant $\mathrm{x}$-ray scattering in general, and so also for this study, is the interference effect. This effect can strongly influence the spectral shape. The requirement for interference to occur is that there are two or more degenerate or quasi-degenerate intermediate core excited states that can be excited coherently, and which can decay to the same final state. The interference between the scattering channels is thus caused by a coherence between different core states simultaneously excited. The coherence and interference between core-excited states is small when the energy gap is small in comparison with $\sqrt{\Gamma^{2}+\Omega^{2}}$ where $\Gamma$ denotes the lifetime of the core excited state and $\Omega$ is the detuning frequency ${ }^{19}$ (difference between resonant and actual excitation frequencies). The intermediate interfering states can also belong to a continuous spectrum, which takes place in solids, for excitation above the ionization threshold or when the core excitation refers to dissociative states.

Theoretical simulations are carried out by taking a repeat unit as a model molecule of the polymer system for PMPV and PDPV; for PPV two repeat units were also tested. Methods for calculations of nonresonant $\mathrm{x}$-ray emission spectra have indeed been implemented in an ab initio band theory framework, see Ref. 20, however, with limitation on the applicable size of the unit cell. In the present case a restriction to one single repeat unit find motivation in the fact that relatively flat bands are obtained for the studied polymer systems. ${ }^{21}$ The geometry of the model molecules were obtained by using the AM1 Hamiltonian, ${ }^{22}$ in the MOPAC program, and periodic boundary conditions. The torsion angle among the phenyl rings, in PPV was assumed to be $7^{\circ}$, as found in a neutron diffraction experiment. ${ }^{23}$ The polymer repeat unit formulae are shown in Fig. 2. The transition dipole moments and the energy levels $(k, n, \nu)$ involved in the cross section formula are obtained from frozen orbital, symmetry delocalized, Hartree-Fock calculations. The separation of energy levels in the spectra are identical to those in the valence levels as measured by ultraviolet photoelectron spectroscopy, while intensities obvisouly are different due to the different physical processes. This holds always for the nonresonant $\mathrm{x}$-ray emission spectra, but also for the resonant $\mathrm{x}$-ray emission spectra as long as the frozen orbital approximation is valid. Assuming this approximation, as we do here, the screening of the occupied levels by the core-excited electron is neglected. The direct SCF program DISCO ${ }^{24}$ modified for STEX calculations ${ }^{25}$ has been employed in orbital energy and dipole transition moment calculations. The simulated results are presented in Figs. 4, 6, and 7 as bar diagrams and as continuous spectra where each bar has been convoluted with a gaussian function with a full width at half maximum of $0.5 \mathrm{eV}$.

\section{RESULTS}

\section{A. Nonresonant inelastic x-ray scattering spectra}

Experimental nonresonant inelastic x-ray scattering spectra (NRIXS) of the poly(phenylenevinylene)s excited with $310 \mathrm{eV}$ photon-energy aligned together with the NEXAFS spectra are presented in Fig. 3. The spectrum of benzene, the main part of which is well understood, ${ }^{26}$ is also 


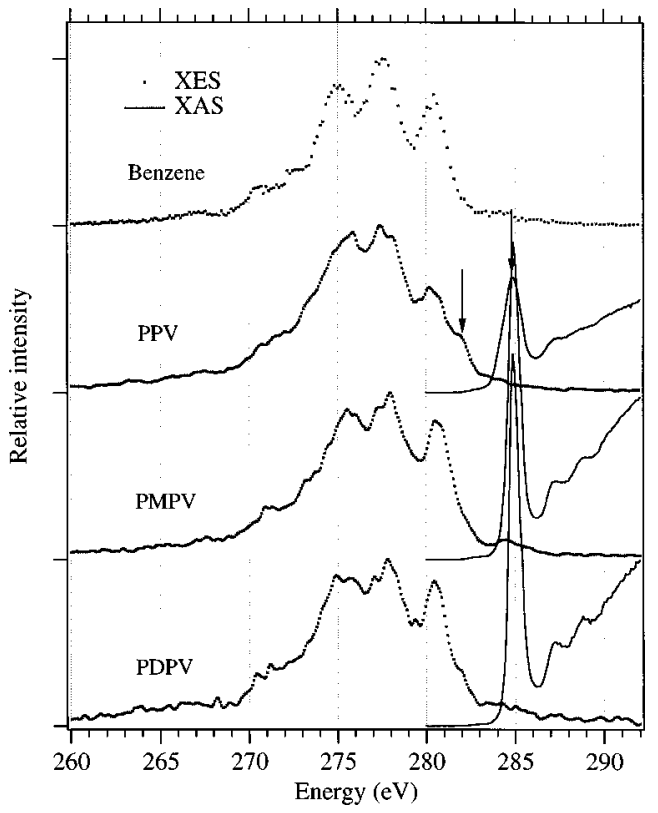

FIG. 3. Experimental nonresonant x-ray scattering spectra excited at 310.0 $\mathrm{eV}$ photon energy aligned together with the absorption spectra.

included for comparison. The energy scales of the spectra have been aligned by using the elastic peak in the RIXS spectra presented later. The detailed discussion of the NEXAFS spectra is planned to be given elsewhere. ${ }^{27}$ The NRIXS spectra of all systems can be grossly subdivided into 6 bands in the energy range from 265 to $283 \mathrm{eV}$. The band patterns of the poly(phenylenevinylene)s resemble those of benzene, and it is therefore relevant to analyze benzene first.

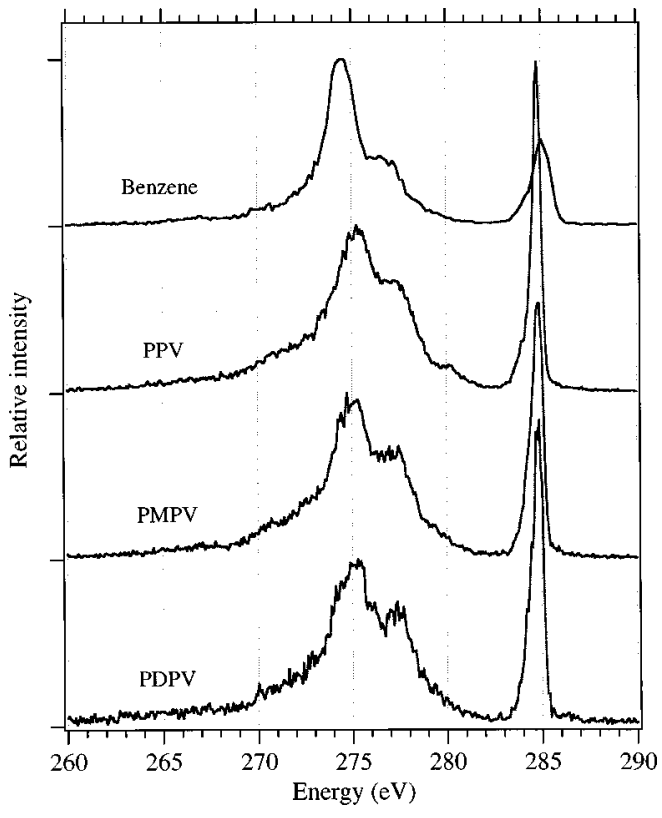

FIG. 5. Experimental resonant x-ray inelastic scattering spectra excited at $284.8 \mathrm{eV}$ photon energy.

Benzene has ground state $D_{6 h}$ symmetry with 9 outer valence MO levels with intensity in the NRIXS spectra. As shown in the bar diagram of Fig. 4 the simulations place these MO's into 6 groups using a binding energy scale $(\varepsilon$ $=I_{1 s}-h \nu$, where $I_{1 s}$ is the $C_{1 s}$ ionization potential and $h \nu$ the photon energy of the emitted x-ray photon). The six bands, which are denoted by the italic letters from $A$ to $F$ from the high energy side, can thus be assigned to x-ray

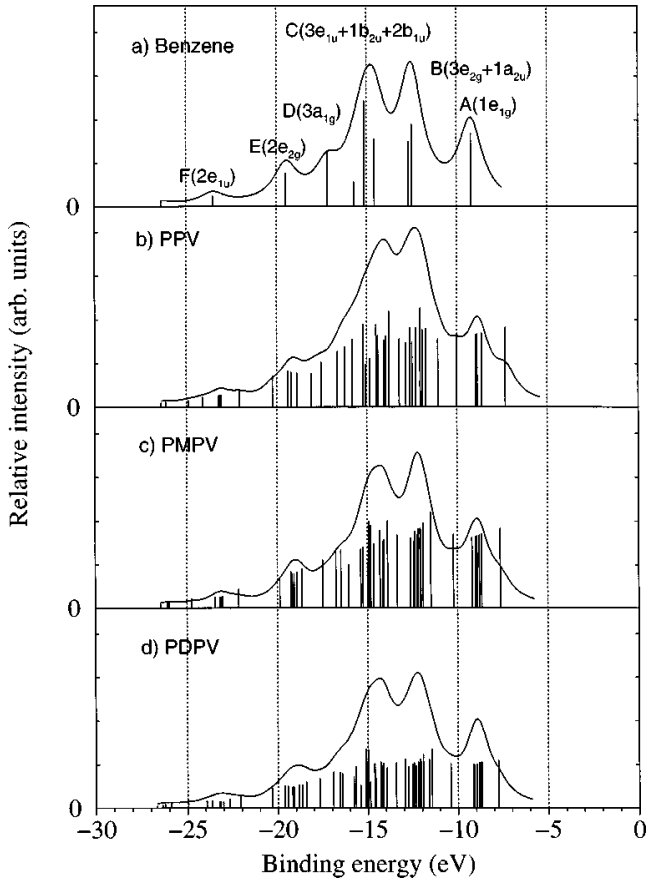

FIG. 4. Calculated nonresonant x-ray scattering spectra of benzene, PPV, PMPV and PDPV. FHWM $=0.5 \mathrm{eV}$ is used in convolution.

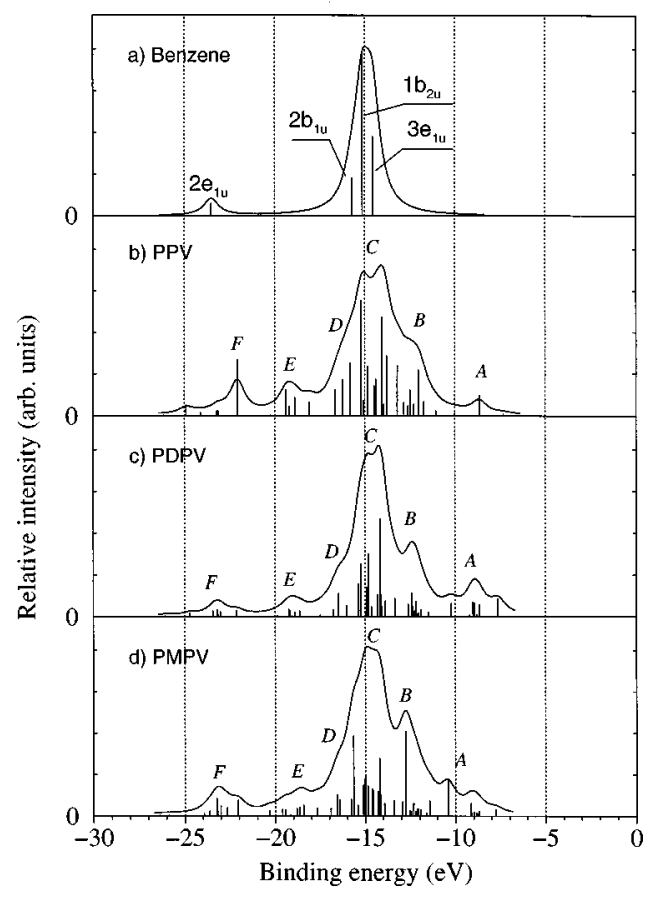

FIG. 6. Calculated resonant $\mathrm{x}$-ray inelastic scattering spectra excited at $284.8 \mathrm{eV}$ photon energy. $\mathrm{FHWM}=0.5 \mathrm{eV}$ is used in convolution. 
TABLE I. Binding energies and functional group population for the $A$ band in PPV.

\begin{tabular}{ccccc}
\hline \hline \multirow{2}{*}{ Band } & \multirow{2}{*}{ Transition } & Binding & \multicolumn{2}{c}{ Population $(\%)$} \\
\cline { 4 - 5 } \multirow{2}{*}{$A$} & energy & phenyl ring & vinylene \\
& 1 & 7.31 & 62.7 & 37.3 \\
& 2 & 9.05 & 65.3 & 34.7 \\
& 3 & 9.35 & 100.0 & 0.0 \\
& 4 & 9.44 & 100.0 & 0.0 \\
& 5 & 10.81 & 53.3 & 46.7 \\
\hline \hline
\end{tabular}

transitions involving the $1 e_{1 g}, 3 e_{2 g}+1 a_{2 u}, 3 e_{1 u}+1 b_{2 u}$ $+2 b_{1 u}, 3 a_{1 g}, 2 e_{2 g}, 2 e_{1 u}$ MO's, respectively. The latter two bands are weak because of the $2 s$ character of the corresponding MOs and presumably also because of the breakdown of the molecular orbital picture with accompanying correlation state splittings. ${ }^{28}$

The NRIXS spectrum of PPV is simulated by considering two repeat units of the polymer, which consists of two phenyl ring and two vinylene subgroups. Population analysis with respect to the phenyl ring and the vinylene groups has been carried out for valence MO's referring to band $A$; see Table I. Transitions from the highest occupied molecular orbital (HOMO) level, which is $\pi$ bonding between phenyl and vinylene, results in an edge structure at the low binding energy (BE) frontier of band $A$. Transitions No. 2, 3 and 4, which correspond to different admixtures of $\pi$ MO's of the phenyl and vinylene groups (see Table I), form band $A$. Similar analysis can be applied to the remaining bands. One finds that the number of transitions with main phenyl character dominate each band, and all transitions have similar intensities within a band. These two features explain the conspicuous resemblance of NRIXS spectra of benzene and PPV.

Similar arguments hold for PMPV and PDPV. Because there are more phenyl rings in the repeat units in PMPV and PDPV (3 and 4 phenyl rings connect with a vinylene group, respectively, see Fig. 2), one can expect an even clearer benzene-like appearance in the NRIXS spectra of these compounds. Results for spectral simulations for the PMPV and PDPV compounds by considering one repeat unit are shown in Fig. 4. They are in good agreement with experiment and also resemble benzene. Going from PPV to PMPV and PDPV, two systematic features can be observed in both experimental and computed spectra. Firstly, the edge structure at the frontier of band $A$ becomes less clear and the relative intensity of band $A$ becomes larger. These changes refer to the enhanced phenyl character of the transitions in the band.

Besides the 6-band structure, there exists a weak feature at the high-energy side of the NRIXS spectrum (at about 284 $\mathrm{eV}$ ) in benzene and in the poly(phenylenevinylene)s. Its high position-about $4 \mathrm{eV}$ higher than the emission energy from the HOMO level in benzene-suggests involvement of a different initial state, namely one in which the core ionization is accompanied by a simultaneous excitation to a virtual MO, i.e. a shake-up transition. Such transitions are known to result in satellite structures both in XPS and NRIXS, in the latter case as an initial state for a valence to core decay.
These so-called Wentzel-Druyvesteyn (WD) satellites ${ }^{29,30}$ have weak intensity due to their multielectron nature, but are nevertheless often visible on the high-energy sides of the main XES spectra.

\section{B. Resonant x-ray inelastic scattering spectra}

The resonant $\mathrm{x}$-ray inelastic scattering spectra (RIXS) were recorded by tuning the incident $\mathrm{X}$-ray photon beam to the first $\pi^{*}$ resonance $(284.8 \mathrm{eV})$ and are presented in Fig. 5. The simulated spectra shown in Fig. 6 are found to agree with the experimental ones. Similarly to the nonresonant case, the resonant spectra of poly(phenylenevinylene)s demonstrate a strong similarity with the resonant spectrum of benzene. Before going through the poly(phenylenevinylene)s, we briefly recapitulate the spectrum of benzene, ${ }^{26}$ then focus on the energy range corresponding to bands $A$ and $B$ of the nonresonant spectrum. The lowest unoccupied molecular orbital (LUMO) is $1 e_{2 u}$ (using ground state $D_{6 h}$ point group symmetry). So when the incident $\mathrm{x}$-ray is tuned to $1 e_{2 u}$, only electrons occupying the ungerade MO's can fill up the $C_{1 s}$ hole MO ( $1 e_{2 g}$ ) according to the parity selection rule for resonant $\mathrm{x}$-ray emission (gerade-gerade and ungerade-ungerade). This assumes that the core excited state maintains the same symmetry as the ground state $\left(D_{6 h}\right)$. In particular, the HOMO $1 e_{1 g}$ level, corresponding to emission energy around $280 \mathrm{eV}$ will be symmetry forbidden. This is the reason that the emission band $A$ of highest energy in the nonresonant spectrum disappears in the resonant spectrum. The band $B$ at $278 \mathrm{eV}$ in the nonresonant spectrum, which originates in emission from the $3 e_{2 g}+1 a_{2 u}$ levels, should also be depleted in the resonant case due to symmetry selection. Although it does show reduced intensity, there is a significant intensity remaining, which was argued as being due to a final state vibronic coupling effect in Ref. 26.

For the poly(phenylenevinylene)s, there exists no symmetry at all, and one could expect from this point of view that the resonant and nonresonant XES spectra would be similar. On the other hand if the vinylene groups exert only small perturbations on the phenyl ring the local electronic structure of the benzene molecule can remain intact and since the x-ray process is local one can then argue that an " "effective", symmetry selection can be restored. The experimental spectra for poly(phenylenevinylene)s indeed show benzene-like features also in the resonant case, with the band $A$ intensity considerably reduced. As seen in Fig. 7 the theoretical simulations implementing the full one-step Kramers-Heisenberg formula but excluding the correlation between transitions (dipole moment of each transition was squared before summation) show non-resonant features with strong intensity for band $A$ (Fig. 4). Thus the restoration of the effective symmetry selection (as well as the benzene similarity) must be ascribed to channel interference; the $\mathrm{x}$ ray scattering of the different close-lying core-hole states interfere in such a way that the total signal is depleted. To estimate the chemical splitting of the $C_{1 s} \rightarrow \pi^{*}$ transitions from the phenyl ring and vinylene core sites, a static exchange (STEX) calculation has been carried out for PPV. It gives a maximum for the energy splittings of $0.18 \mathrm{eV}$ for the $C_{1 s} \rightarrow \pi^{*}$ excitations corresponding to different core sites 


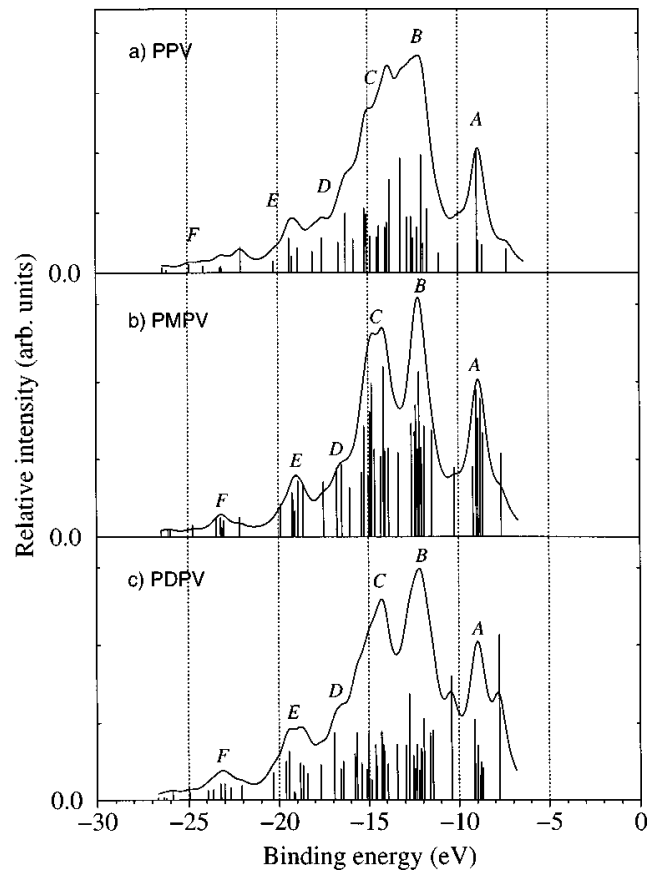

FIG. 7. Calculated resonant $x$-ray inelastic scattering spectra excited at $284.8 \mathrm{eV}$ photon energy, not including interference effects. $\mathrm{FHWM}=0.5 \mathrm{eV}$ is used in convolution.

(actually also for the $C_{1 s}$ ionization potentials). Such a small value is comparable with the lifetime broadening of the core excited states and explains the strong interference effects.

Similar arguments hold for the band $B$. It is the most intense band in the XE simulations, but reduces considerably for the resonant case, provided interference is taken into account. It refers to the $3 e_{2 g}$ emission which is symmetry forbidden in benzene, but has probably $a_{2 u}$ derived intensity, as well, which is forbidden but not nonzero in benzene (see above). Band $C$ has contributions mostly from the MO's of the phenyl ring which are symmetry allowed in the $D_{6 h}$ group representation. It comes out as the most intensive band in the resonant spectra of the poly-phenylene-vinylene compounds.

Geometry optimizations of the compounds indicate small changes of the internal benzene geometries. As mentioned above we find small chemical shifts in $C_{1 s} \rightarrow \pi^{*}$ excitation energies corresponding to different localized carbon sites. The XE spectra are fairly insensitive to the actual geometries (which agrees with the obvious fact that the spectra of the different compounds are quite similar). The RIXS spectra are more geometry-and electronic structure- dependent in that the interference effects become weakened if the chemical shifts increase.

\section{DISCUSSION}

The present results have a strong tie to both the symmetry selection observed in molecules and the famous momentum conservation rule for incoming and scattered photons in solid state RIXS. ${ }^{17,31-33}$ The latter approximation holds in the frozen orbital approximation and rests on the interference effect; when the interference of the RIXS channels through core hole states on different atoms is neglected then momen- tum conservation breaks down. This leads to a strong dependence of the RIXS spectral shape on the excitation energy, which has been confirmed by several experiments. ${ }^{34-36}$ The selection rules for the RIXS tensor in molecular systems, ${ }^{12}$ observed in many cases, ${ }^{37}$ constitute the molecular analog of the momentum conservation law. As theoretically predicted in Refs. 17 and 33 this law implies a shift of the threshold for RIXS of alkali metals and in $\pi$-electron systems, and predicts zero RIXS intensity for occupied $\pi$ bands in $\pi$-electron systems, which is very well confirmed in the present work. A simple explanation of this fact is given in Ref. 33, which shows the important role of interference for this effect and that this "vanished" RIXS intensity is transferred to the elastic cross section, which is not equal to zero in the forbidden region.

The electron-photon, electron-phonon and electron-corehole interactions violate the momentum conservation law and allow emission in the forbidden $\pi$ region. Although the present compounds are not pure $\pi$-electron systems one can still regard the amount of intensity in the "forbidden" $A$ band region as an indication of the strength of these interactions. As shown recently, ${ }^{38,39}$ the effective strength of the electron-phonon interaction can be significantly reduced even by small detuning of the incident $\mathrm{x}$-ray photon frequency below the absorption band.

We note that by combining the resonant and the nonresonant $\mathrm{XE}$ spectra, one can obtain the band gap of the compound. It equals the energy difference between the elastic peak in the RIXS spectrum and the front edge of the nonresonant XE spectrum of the experimental spectra. They are close to $2.8 \mathrm{eV}$ for all the PPV, PMPV, and PDPV polymers. These quantities seem to fall in between the values obtained from optical absorption spectroscopy, ${ }^{40} 2.45 \mathrm{eV}$ for PPV, and ultraviolet photoelectron spectroscopy $3.1 \mathrm{eV}$ for PPV. ${ }^{41}$ The differences might refer to the different broadening mechanisms for the bands and to the actual identification of the adiabatic or vertical transition energies which might come out differently in the different kinds of spectroscopies.

\section{SUMMARY}

Resonant and nonresonant x-ray scattering spectra of various poly(phenylenevinylene)s have been measured with monochromatic synchrotron radiation. As in benzene and other molecular organic systems, ${ }^{42,26,43,44}$ the analysis based on $a b$ initio canonical Hartree-Fock theory reproduced the main features of the poly(phenylenevinylene)s. The conspicuous resemblance with benzene in both resonant and nonresonant spectra indicates no major electronic or geometric structure changes in the phenyl rings connecting with a vinylene group, and indicates also that the phenyl ring works as an excellent building block for these spectra. The benzene-like features in the RIXS spectra of the poly(phenylenevinylene)s are interpreted as the result of a strong channel interference. Only by accounting for this interference the $\pi$-electron emission derived from the forbidden $e_{1 g}$ level in benzene vanishes for the polymers. The connection with the momentum conservation law was pointed out, and that the remaining intensity observed should be taken as an indication of the interactions not accounted for in the frozen 
orbital approximation - the electron-photon, electron-phonon and electron-core-hole interactions-and which also breaks the momentum conservation rule. ${ }^{33}$

Two other aspects were presented and discussed in the paper, namely that the edges of the occupied bands in each case could be identified in the nonresonant spectra, and that the subtraction of the measured core-excitation (LUMO resonance) and nonresonant HOMO emission energies give an alternative way to determine the optical band gap. Both these aspects, as well as the building block character and the particular interference effects here studied, might be worth exploiting for other types of polymers as possible diagnostic tools.

\section{ACKNOWLEDGMENTS}

This work was supported by the Swedish Natural Science Research Council (NFR), the Göran Gustavsson Foundation for Research in Natural Sciences and Medicine and the Swedish Institute (SI). The experimental work at ALS, Lawrence Berkeley National Laboratory was supported by the director, Office of Energy Research, Office of Basic Energy Sciences, Materials Sciences Division of the U. S. Department of Energy, under Contract No. DE-AC0376SF00098. J.-H.G. gratefully acknowledges financial support from the NFR. L.Y. would like to acknowledge the support from National Natural Science Foundation of China, and R.D. support from the National University of Malaysia.

${ }^{1}$ C. K. Chiang, C. R. Fincher, Y. W. Park, A. J. Heeger, H. Shirakawa, E. J. Louis, S. C. Gau, and A. G. MacDiarmid Phys. Rev. Lett. 39, 1098 (1977).

${ }^{2}$ J. H. Burroughes, D. D. C. Bradley, A. R. Brown, R. N. Marks, K. Mackay, R. H. Friend, P. L. Burns, and A. P. Holmes, Nature (London) 347, 539 (1990).

${ }^{3}$ J. H. Burroughes, C. A. Jones, and R. H. Friend, Nature (London) 335, 137 (1988).

${ }^{4}$ F. Garnier, G. Horowitz, X. Peng, and D. Fichou, Adv. Mater. 2, 592 (1990).

${ }^{5}$ H. M. O’Bryan and H. W. B. Skinner, Phys. Rev. 45, 370 (1934).

${ }^{6}$ H. Jones, N. F. Mott, and H. W. B. Skinner, Phys. Rev. 45, 379 (1934).

${ }^{7}$ T. Warwick, P. Heimann, D. Mossessian, W. McKinney, and H. Padmore, Rev. Sci. Instrum. 66, 2037 (1995).

${ }^{8}$ J. Nordgren and R. Nyholm, Nucl. Instrum. Methods A 246, 242 (1986).

${ }^{9}$ P. L. Burn, D. D. C. Bradley, R. H. Friend, D. A. Haliday, A. B. Holmes, R. W. Jackson, and A. M. Kraft, J. Chem. Soc. Perkin Trans. 1, 3225 (1992).

${ }^{10}$ W. J. Feast and I. S. Millichamp, Polym. Commun. 24, 102 (1983).

${ }^{11}$ F. Cacialli, R. Daik, P. Dounis, W. J. Feast, R. H. Friend, N. D. Haylett, C. P. Jarrett, C. Schoenenberger, J. A. Stevens, and G. Widawski, Philos. Trans. R. Soc. London, Ser. A 355, 707 (1997).
${ }^{12}$ F. Kh. Gel'mukhanov and H. Ågren, Phys. Rev. A 49, 4378 (1994).

${ }^{13}$ F. Kh. Gel'mukhanov, L. N. Mazalov, and N. A. Shklyaeva, Sov. Phys. JETP 42, 1001 (1975)

${ }^{14}$ T. Åberg, Phys. Scr. 21, 495 (1980).

${ }^{15}$ Y. Luo, H. Ågren, and F. K. Gelmukhanov, Phys. Rev. A 53, 1340 (1996).

${ }^{16}$ Advanced Quantum Mechanics (Addison-Wesley, Reading, MA, 1997), Chap. 2.

${ }^{17}$ F. Kh. Gel'mukhanov, L. N. Mazalov, and A. V. Kontratenko, Chem. Phys. Lett. 46, 133 (1977).

${ }^{18}$ Y. Luo, H. Ågren, and F. Gel'mulhanov, J. Phys B: At. Mol. Phys. 27, 4169 (1994).

${ }^{19}$ F. Gel'mukhanov, T. Privalov, and H. Ågren, Phys. Rev. A 56, 256 (1997)

${ }^{20}$ C. M. Liegener and H. Ågren, J. Chem. Phys. 99, 2821 (1993).

${ }^{21}$ M. Lögdlund, W. R. Salaneck, F. Meyers, J. L Bredas, G. A. Arbuckle, R. H. Frined, A. B. Holmes, and G. Froyer, Macromolecules 26, 1959 (1993).

${ }^{22}$ M. J. S. Dewar, E. G. Zoebisch, E. F. Healy, and J. J. P. Stewart, J. Am. Chem. Soc. 107, 3092 (1985).

${ }^{23}$ G. Mao, J. E. Fischer, F. E. Karasz, and M. J. Winokur, J. Chem. Phys. 98, 712 (1993).

${ }^{24}$ J. Almlöf, K. Faegri, Jr., and K. Korsell, J. Comput. Chem. 3, 385 (1982).

${ }^{25}$ H. Ågren, V. Carravetta, O. Vahtras, and L. G. M. Pettersson, Chem. Phys. Lett. 222, 75 (1994).

${ }^{26}$ P. Skytt, J. H. Guo, N. Wassdahl, J. Nordgren, Y. Luo, and H. Ågren, Phys. Rev. A 52, 3572 (1995).

${ }^{27}$ J.-H. Guo, M. Magnuson, C. Såthe, A. Agui, J. Nordgren, L. Yang, Y. Luo, and H. Ågren (unpublished).

${ }^{28}$ L. S. Cederbaum, W. Domcke, J. Schirmer, W. von Niessen, G. H. F. Diercksen, and W. P. Kraemer, J. Chem. Phys. 69, 1591 (1978).

${ }^{29}$ G. Wentzel, Z. Phys. 31, 445 (1925).

${ }^{30}$ M. J. Druyvesteyn, Z. Phys. 43, 707 (1927).

${ }^{31}$ Y. Ma, Phys. Rev. B 49, 5799 (1994).

${ }^{32}$ Y. Ma and M. Blume, Rev. Sci. Instrum. 66, 1543 (1995).

${ }^{33}$ F. Gel'mukhanov and H. Ågren, Phys. Rev. B 57, 2780 (1998).

${ }^{34}$ Y. Ma, P. Skytt, N. Wassdahl, P. Glans, D. C. Mancini, J. H. Guo, and J. Nordgren, Phys. Rev. Lett. 71, 3725 (1993).

${ }^{35}$ Y. Ma, K. E. Miyano, P. L. Cowan, Y. Agliakiy, and B. A. Karlin, Phys. Rev. Lett. 74, 478 (1995).

${ }^{36}$ J. A. Carlisle, E. L. Shirley, E. A. Hudson, L. J. Terminello, T. A. Callcott, J. J. Jia, D. L. Ederer, R. C. C. Perera, and F. J. Himpsel, Phys. Rev. Lett. 74, 1234 (1995).

${ }^{37}$ J. Nordgren, J. Electron Spectrosc. Relat. Phenom. 78, 25 (1996).

${ }^{38}$ P. Skytt, P. Glans, J.-H. Guo, K. Gunnelin, J. Nordgren, F. Gel'mukhanov, A. Cesar, and H. Ågren, Phys. Rev. Lett. 77, 5035 (1996).

${ }^{39}$ A. Cesar, F. Gel'mukhanov, Y. Luo, H. Ågren, P. Skytt, P. Glans, J.-H. Guo, K. Gunnelin, and J. Nordgren, J. Chem. Phys. 106, 3439 (1997).

${ }^{40}$ J. L. Bredas, J. Cornil, and A. J. Heeger, Adv. Mater. 8, 447 (1996).

${ }^{41}$ M. Fahlman, M. Lögdlund, S. Stafström, W. R. Salaneck, R. H. Friend, P. L. Burn, A. B. Holmes, K. Keriyama, Y. Sonoda, O. Lhost, F. Meyers, and J. L Bredas, Macromolecules 28, 1959 (1995).

${ }^{42}$ Y. Luo, H. Ågren, F. Gel'mulhanov, J. H. Guo, P. Skytt, N. Wassdahl, and J. Nordgren, Phys. Rev. B 52, 14479 (1995).

${ }^{43}$ Y. Luo, H. Ågren, J. H. Guo, P. Skytt, N. Wassdahl, and J. Nordgren, Phys. Rev. A 52, 3730 (1995).

${ }^{44}$ J. H. Guo, P. Skytt, N. Wassdahl, J. Nordgren, Y. Luo, O. Vahtras, and H. Ågren, Chem. Phys. Lett. 245, 152 (1995). 